\title{
Saisonnalité de la production laitière bovine et implications pour le renforcement de la collecte industrielle dans la wilaya de Skikda (Algérie)
}

\author{
Karima Zalani ${ }^{1}$ Guillaume Duteurtre ${ }^{2}$ \\ Mohammed Tahar Benyoucef ${ }^{3 *}$
}

\begin{abstract}
Mots-clés
Bovin, lait cru, exploitation laitière, profil, industrie laitière, système d'exploitation agricole, Algérie
\end{abstract}

Submitted: 29 July 2020

Accepted: 15 February 2021

Published: 30 June 2021

DOI: $10.19182 /$ remvt.36365

\begin{abstract}
Résumé
Une étude sur la filière laitière dans la wilaya de Skikda (Algérie) a permis d'analyser la saisonnalité de la production laitière chez 157 exploitations bovines et les implications relatives à la collecte de lait par l'industrie. Les enquêtes ont porté sur les deux systèmes de production (avec terre et hors-sol) en 2016 et 2017 pour évaluer l'importance des contributions des systèmes d'exploitation dans l'approvisionnement des laiteries. Une première analyse en composantes principales a mis en évidence quatre profils de livraison. Une deuxième analyse effectuée sur les vêlages observés dans les troupeaux des exploitations montre une certaine concordance entre la saisonnalité des livraisons de lait et la répartition des vêlages des troupeaux. L'étude a aussi abordé les notions de territoires et de distances parcourues pour les livraisons du lait aux laiteries. Certaines exploitations livraient leur lait à deux laiteries implantées à Skikda qui avaient aussi recours à un approvisionnement en poudre de lait à des prix subventionnés. D'autres livraient leur lait à des centres de collecte de lait implantés dans le bassin laitier et appartenant à deux laiteries industrielles localisées à plus de 250 kilomètres en moyenne dans la wilaya de Bejaia. Ces dernières s'approvisionnaient aussi en poudre de lait mais aux prix libres sur le marché. Elles mettaient en œuvre des dispositifs d'appui aux éleveurs qui permettaient de diversifier leur approvisionnement afin de lisser les quantités collectées et de compenser la saisonnalité individuelle des fermes. Des dispositifs de concertations pourraient permettre à ces industriels d'accompagner les éleveurs dans des stratégies plus explicites de dé-saisonnement de la production.
\end{abstract}

- Comment citer cet article : Zalani K., Duteurtre G., Benyoucef M.T., 2021. Seasonality of bovine milk production and implications for the reinforcement of industrial collection in Skikda Wilaya (Algeria). Rev. Elev. Med. Vet. Pays Trop., 74 (2): 83-92, doi: 10.19182/remvt.36365

\section{INTRODUCTION}

En Algérie, la politique laitière se caractérise par le recours à des importations massives de poudre de lait pour l'approvisionnement des laiteries conventionnées avec l'Office national interprofessionnel du

\footnotetext{
1. Département d'agronomie, Université 20 août 1955, Skikda, Algérie. 2. CIRAD, UMR SELMET, F-34398 Montpellier, France ; SELMET, Univ Montpellier, CIRAD, INRAE, Institut Agro, Montpellier, France 3. Département production animale, Ecole nationale supérieure agronomique, Alger, Algérie.

* Auteur pour la correspondance Email : mtbenyoucef@hotmail.fr
}

lait et de produits laitiers (ONIL). Cet office est chargé par les pouvoirs publics de la régulation du marché dans le cadre d'une politique de subvention des prix à la consommation de lait pasteurisé et conditionné en sachets (LPS). Il importe les poudres de lait aux prix du marché international et les revend à un prix subventionné aux industriels avec lesquels il est conventionné. Le prix au détail du litre de LPS est administré et fixé à 25 dinars (DA). Les industriels produisant du lait conditionné en briques et des produits laitiers ne bénéficient pas de ces subventions mais importent eux aussi massivement de la poudre de lait (Benyoucef et al., 2013). La facture de ces importations laitières, déjà très élevée au début des années 2000 (Benyoucef, 2005), n'a cessé de croître pour atteindre, en 2017, 3,4 millions de tonnes équivalents lait soit 1,4 milliard de dollars US (FAO, 2020). Cette politique de subvention des prix à la consommation du lait pasteurisé standard et de recours au marché international en poudre de 
lait a permis de répondre à la forte demande en lait des populations urbaines. Entre 1973 et 2013, la consommation algérienne de produits laitiers s'est envolée, passant de 50 à 141 kilogrammes équivalents laits par habitant et par an. La forte demande en lait de consommation des centres urbains a ainsi maintenu le pays dans une certaine dépendance vis-à-vis du marché international des poudres de lait (Sraïri et al., 2013).

Dans le même temps, l'Etat a poursuivi un objectif de développement de l'élevage laitier domestique destiné à contribuer au développement agricole local. Le programme national de réhabilitation de la filière laitière locale lancé en 1995 et les programmes laitiers suivants ont permis de mettre en place divers soutiens financiers destinés à favoriser la collecte de lait local par les laiteries. Ces soutiens incluaient au moment des enquêtes une prime aux producteurs (12 DA/L), une prime aux collecteurs (5 DA/L) et une prime d'intégration du lait cru aux industriels variant de 4 à 7,5 DA/L (Kheffache et Tozanli, 2015). Or, le renforcement de la collecte de lait local par les laiteries souffre de la faible coordination entre les producteurs et les autres acteurs de la filière laitière locale (Hadji Kouidri et al., 2018). Les performances de collecte et de transformation du lait cru sont variables du fait du grand nombre d'acteurs dont les relations contractuelles restent à consolider dans le contexte d'une économie de marché (Sraïri et al., 2013 ; Benyoucef, 2017). Cette situation se traduit par une intégration difficile du lait local dans l'industrie, un développement continu des ventes de lait informelles et une forte dépendance de l'Algérie visà-vis du marché international du lait en poudre. Les performances de collecte de lait réalisées ont certes augmenté pour atteindre 893 millions de litres en 2016. Les laiteries privées conventionnées avec l'ONIL assurent $68 \%$ de ce volume de lait grâce aux subventions de l'Etat à partir de 2008. Cependant, ce niveau de collecte de lait reste insuffisant eu égard aux potentialités locales de production des bassins laitiers. Il s'explique aussi par une profession de producteurs inorganisés et faiblement contractualisés avec des laiteries qui continuent à privilégier un approvisionnement mixte composé de poudre de lait importées et de lait local collecté (Benyoucef, 2017).

Pour mieux comprendre ces blocages dans les systèmes de collecte de lait, l'étude s'interroge sur l'importance de la saisonnalité de la collecte de lait cru dans la wilaya de Skikda. Plusieurs travaux réalisés dans d'autres pays ont en effet souligné les contraintes que représentent les irrégularités saisonnières de la production de lait dans la mise en place de systèmes de collecte pérennes (Dieye et al., 2005 ; 2008 ; Duteurtre, 2007 ; Losq et al., 2007 ; Delmotte et al., 2008 ; Napoléone, 2019; Gaye et al., 2020). Ces contraintes sont particulièrement prégnantes en Algérie où la production fourragère est fortement saisonnière tandis que la consommation de lait augmente beaucoup à d'autres périodes de l'année, notamment pendant le mois de Ramadan (Kheffache et Tozanli, 2015 ; Hadji Kouidri et al., 2018). Notre étude vise à caractériser les profils de livraison de lait d'exploitations enquêtées dans le contexte de Skikda. Elle met l'accent également sur une analyse des profils des vêlages des troupeaux de ces mêmes exploitations afin de chercher à comprendre si la répartition de leurs vêlages expliquerait la saisonnalité de leurs livraisons de lait selon le système de production pratiqué. En raison de l'émergence d'élevages laitiers hors-sol, l'étude a tenté d'établir des typologies distinctes pour chaque système d'exploitation (avec terre et hors-sol) sur des données de livraisons mensuelles de lait dans le but d'apprécier l'importance de leur contribution dans l'approvisionnement des laiteries. L'étude traite également des aspects territoriaux et de distances parcourues pour la collecte du lait par les laiteries. Il s'agit aussi d'apporter une contribution dans la recherche d'une nouvelle dynamique de la collecte de lait par une articulation coordonnée du segment production fermière avec celui de la transformation en vue d'assurer une meilleure intégration industrielle du lait cru dans le développement de l'économie laitière locale.

\section{MATERIEL ET METHODES}

L'étude de la saisonnalité de la collecte de lait cru s'inscrit dans un travail de recherche qui porte sur différentes wilayas (départements) en Algérie dont celle de Skikda. Son effectif bovin laitier est estimé à 82140 vaches dont 67100 vaches de races locales et croisées (81,7\%) et 15040 vaches de races laitières sélectionnées $(18,3 \%)$. Elle a été réalisée à travers un suivi de 157 exploitations bovines laitières de Skikda pendant deux ans, de janvier 2016 à décembre 2017. Ces exploitations pratiquaient l'élevage bovin laitier en système de production avec terre (AT) ou hors-sol (HS). L'échantillon d'exploitations a été sélectionné de manière aléatoire parmi les exploitations de 21 communes de Skikda. $\mathrm{Au}$ total, le troupeau des exploitations suivies se composait d'un effectif de 1324 vaches laitières dont la composition était dominée par la race pure Holstein $(51,1 \%)$. Le reste était réparti entre d'autres races pures dont la Frisonne Pie noir (34,7 \%), la Montbéliarde (10,0 \%), la Fleckvieh, la Normande et la Brune des Alpes $(2,2 \%)$, et les races locales et métisses $(2,0 \%)$.

Les exploitations laitières se caractérisaient par une très grande variabilité de leurs superficies. La surface agricole utilisée des exploitations avec terre $(n=121)$ était en moyenne de 7,7 ha avec une variation de 1 à 38 ha par exploitation, alors que la surface fourragère moyenne était de 5,6 ha par exploitation. Le nombre moyen de vaches laitières était de 8,4 têtes par exploitation. Quant aux exploitations hors-sol $(n=36)$, elles achetaient des fourrages sur le marché ou louaient sur pied des surfaces fourragères dont la moyenne était de 3,3 ha par exploitation. Le nombre moyen de vaches laitières était de 7,0 par exploitation.

Les données mensuelles des livraisons de lait des exploitations ont été recueillies auprès de toutes les laiteries collectant le lait de Skikda. Il s'agissait de Sahlait (A) et Saplait (B) implantées à Skikda, et des laiteries industrielles Soummam (C) et Danone (D) localisées dans la wilaya de Bejaïa à une distance de plus de 200 kilomètres du bassin laitier de collecte.

Ces données ont ensuite été exprimées en pourcentages mensuels qui ont été utilisés pour établir une typologie dans le but de déterminer des groupes d'élevages ayant des profils de livraison voisins, caractérisés par une saison de production maximale. Ensuite, le poids dans les livraisons de chaque type de profil a été estimé en termes de volumes réels de lait livrés dans la collecte globale. Les données mensuelles présentées pour les 12 mois de l'année étaient une moyenne des années 2016 et 2017. Cette valeur moyenne a été prise en considération car la variabilité interannuelle a été très faible dans la zone d'étude (valeurs proches des précipitations annuelles, respectivement en 2016 et 2017 de $737,6 \mathrm{~mm}$ et $765,6 \mathrm{~mm}$, et des températures annuelles moyennes de $19,1^{\circ} \mathrm{C}$ et $19,0^{\circ} \mathrm{C}$ ). En outre, les paramètres structurels (superficies agricoles et effectifs bovins) des exploitations suivies étaient stables au cours des deux années. De même, la stratégie adoptée dans ces exploitations n'a pas changé au cours des deux années de suivi (modes de conduites alimentaire et sanitaire des troupeaux bovins laitiers).

Les données mensuelles de vêlage de ces exploitations ont été recueillies par suivi auprès des chefs d'exploitation et ont servi à établir une typologie des vêlages de leurs troupeaux bovins au cours de la même période, de janvier 2016 à décembre 2017. L'établissement de ces deux typologies par système de production (profils de livraison et profils de vêlage) a été réalisé à l'aide du logiciel Statistica sur les 12 variables mensuelles respectives par une analyse en composantes principales (ACP) suivie d'une classification hiérarchique ascendante. Afin de prendre en compte les différences structurelles très fortes entre les exploitations avec terre (qui pratiquaient des cultures fourragères) et les exploitations sans terre (qui achetaient la totalité de leur alimentation), les résultats ont été présentés séparément pour ces deux types d'exploitation.

Des données qualitatives ont été recueillies par enquête auprès des laiteries A et B de Skikda, et des laiteries C et D de Bejaia dans le but 
de comprendre leurs stratégies de collecte et leurs relations avec les éleveurs laitiers (choix des élevages laitiers, niveau d'intervention et de soutien au développement de la production laitière).

\section{RESULTATS}

\section{Répartition des livraisons moyennes mensuelles de lait de l'ensemble des exploitations}

A Skikda, les moyennes mensuelles des livraisons de lait par exploitation ont montré un pic en mai $(11,0 \%)$ et une baisse sensible en automne (figure 1). Au cours du suivi, la valeur moyenne la plus faible a été observée en novembre représentant seulement 6,8 \% des livraisons annuelles de l'ensemble des exploitations suivies. Le ratio moyen de saisonnalité (défini par le volume de lait livré en période de haute livraison rapporté au volume de lait livré en période de basse livraison) s'est établi à 1,62 pour l'ensemble des exploitations (variation de 1,3 à 2,8 ) dans les réseaux de collecte de lait de Skikda.

Le pourcentage optimal de collecte de lait et de vêlage par mois a été de 8,3\%. Ainsi le pourcentage mensuel était considéré comme important s'il était supérieur à cette valeur. Cette observation rejoint celle de Losq (2007) dans une étude sur la répartition des vêlages d'exploitations livrant du lait à des laiteries en Bretagne (France).

\section{Types de profils de livraison de lait en système d'exploitation avec terre}

Les trois premiers axes factoriels de l'ACP appliquée aux profils de livraison des exploitations avec terre ont expliqué respectivement $35,8 \%, 23,0 \%$ et 10,4 \%, de l'inertie totale, soit un total de 69,2\% (figure 2a). Cette analyse a mis en évidence quatre types de profils de livraison (figure $2 \mathrm{~b}$; tableau I) :

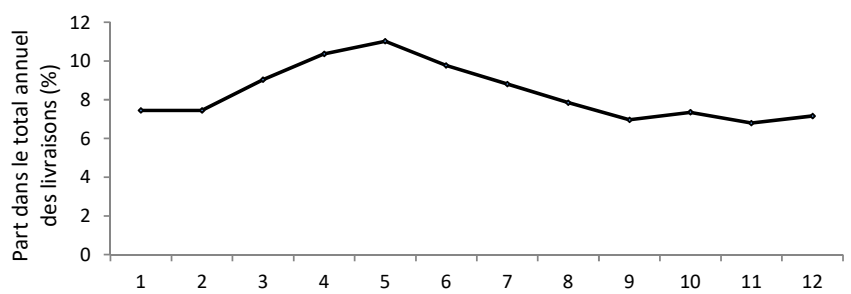

Figure 1 : Répartition des moyennes des livraisons de lait des exploitations $(\mathrm{n}=157)$ selon le mois (Algérie) /// Distribution of average milk deliveries of farms $(n=157)$ by month (Algeria)
- profil ATL1, livraisons très saisonnées de printemps $(\mathrm{n}=38)$. Ces exploitations présentaient un pic de livraison en milieu de printemps (avec un maximum moyen de 13,2 \% du total des livraisons en mai) et une baisse très forte des livraisons en automne (avec un minimum de 5,0 \% du total des livraisons en novembre);

- profil ATL2, livraisons moyennement saisonnées et tardives $(n=22)$. Ces exploitations avaient un pic de livraisons marqué en fin de printemps et au début de l'été (12,3\% en juin) et une baisse très forte des livraisons en automne et en hiver (avec un minimum de $5,9 \%$ en décembre) ;

- profil ATL3, livraisons faiblement saisonnées et précoces $(n=27)$. Ces exploitations se caractérisaient par un très léger pic au début du printemps (11\% du total des livraisons en avril) et une baisse sensible en automne (6,5\% en septembre) ;

- profil (ATL4), livraisons étalées sur toute l'année $(n=34)$, à hauteur de 8 à $9 \%$ de livraisons mensuelles.

\section{Types de profils de livraison de lait en système d'exploitation hors-sol}

Les trois premiers axes factoriels de l'ACP appliquée aux profils de livraison des exploitations hors-sol $(n=36)$ ont expliqué respectivement $33,80 \%, 29,25 \%$ et $11,75 \%$ de l'inertie totale, soit un total de $74,8 \%$ (figures $3 \mathrm{a}$ ). L'analyse a abouti à trois types de profils de livraison (figure $3 b)$ :

- profil HSL1, livraisons très saisonnées de printemps $(n=9)$. Ces exploitations se distinguaient par une forte proportion de livraisons au printemps (15,6\% en mai) et une forte chute en automne (seulement $4,6 \%$ en novembre) ;

- profil HSL2, livraisons moyennement saisonnées et tardives $(n=3)$.

Ces exploitations se caractérisaient par un pic de livraisons au début

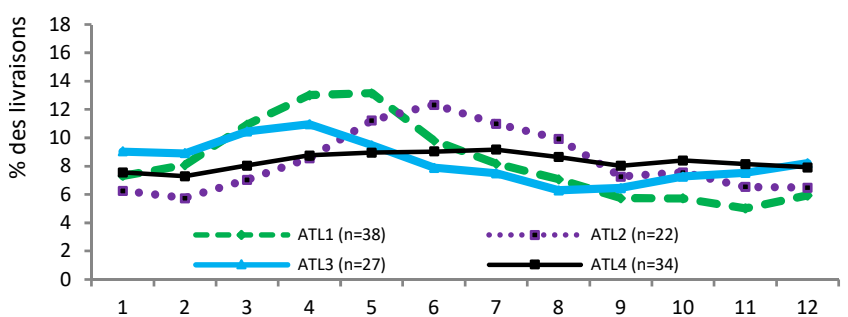

Figure $\mathbf{2 b}$ : Types de profils de livraison des exploitations laitières avec terre selon le mois (Algérie) //I Types of delivery profiles of dairy farms with land by month (Algeria)
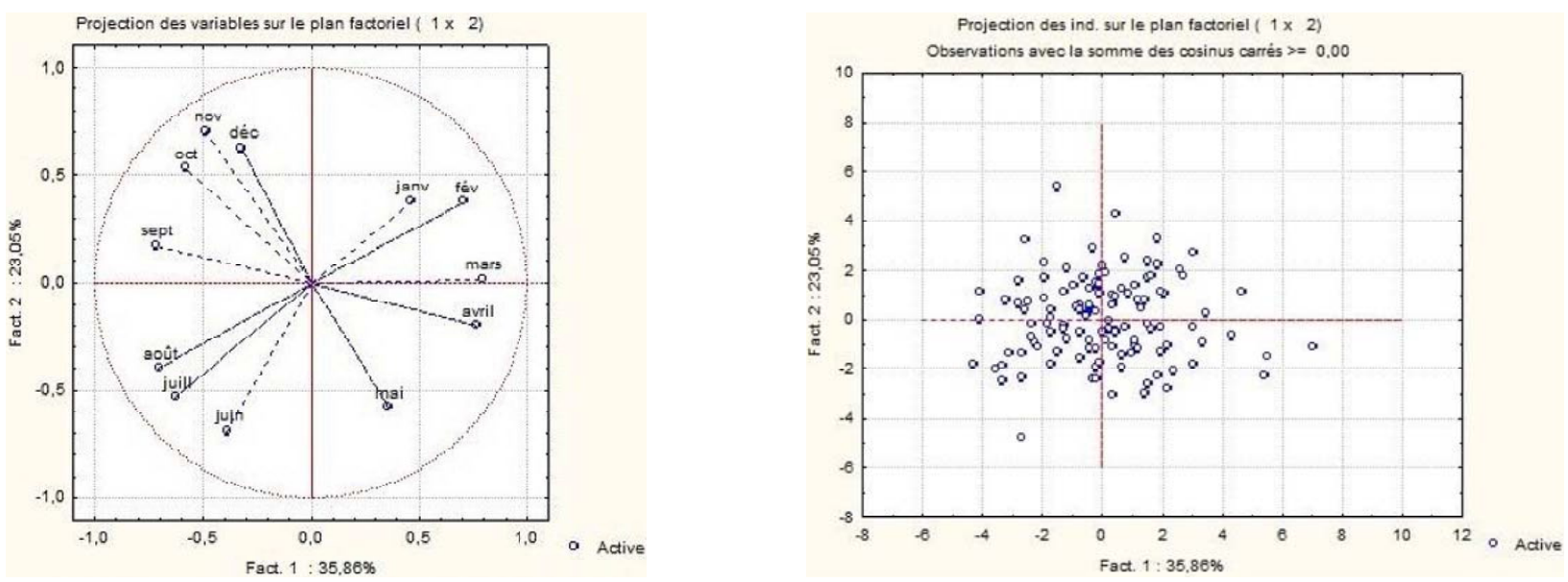

Figure 2a : Représentation et projection des variables et des exploitations laitières avec terre $(n=121)$ sur les deux premiers axes factoriels (Algérie) /// Graph and projection of variables and dairy farms with land $(n=121)$ on the first two factorial axes (Algeria) 
Tableau I : Répartition des exploitations laitières par système et profil de livraison (Algérie) //I Distribution of dairy farms by system and delivery profile (Algeria)

\begin{tabular}{lccccr}
$\begin{array}{l}\text { Système } \\
\text { d'exploitation }\end{array}$ & $\begin{array}{c}\text { Saisonnée printemps } \\
\text { Nb. (\%) }\end{array}$ & $\begin{array}{c}\text { Tardive } \\
\text { Nb. }(\%)\end{array}$ & $\begin{array}{c}\text { Précoce } \\
\text { Nb. }(\%)\end{array}$ & $\begin{array}{c}\text { Etalée sur l'année } \\
\text { Nb. }(\%)\end{array}$ & $\begin{array}{c}\text { Total } \\
\text { Nb. }(\%)\end{array}$ \\
\hline Avec terre & $38(31,4)$ & $22(18,2)$ & $27(22,3)$ & $34(28,1)$ & $121(100)$ \\
Hors-sol & $9(25,0)$ & $3(8,3)$ & - & $24(66,7)$ & $36(100)$ \\
Total & $47(29,9)$ & $25(15,9)$ & $27(17,2)$ & $58(36,9)$ & $157(100)$
\end{tabular}
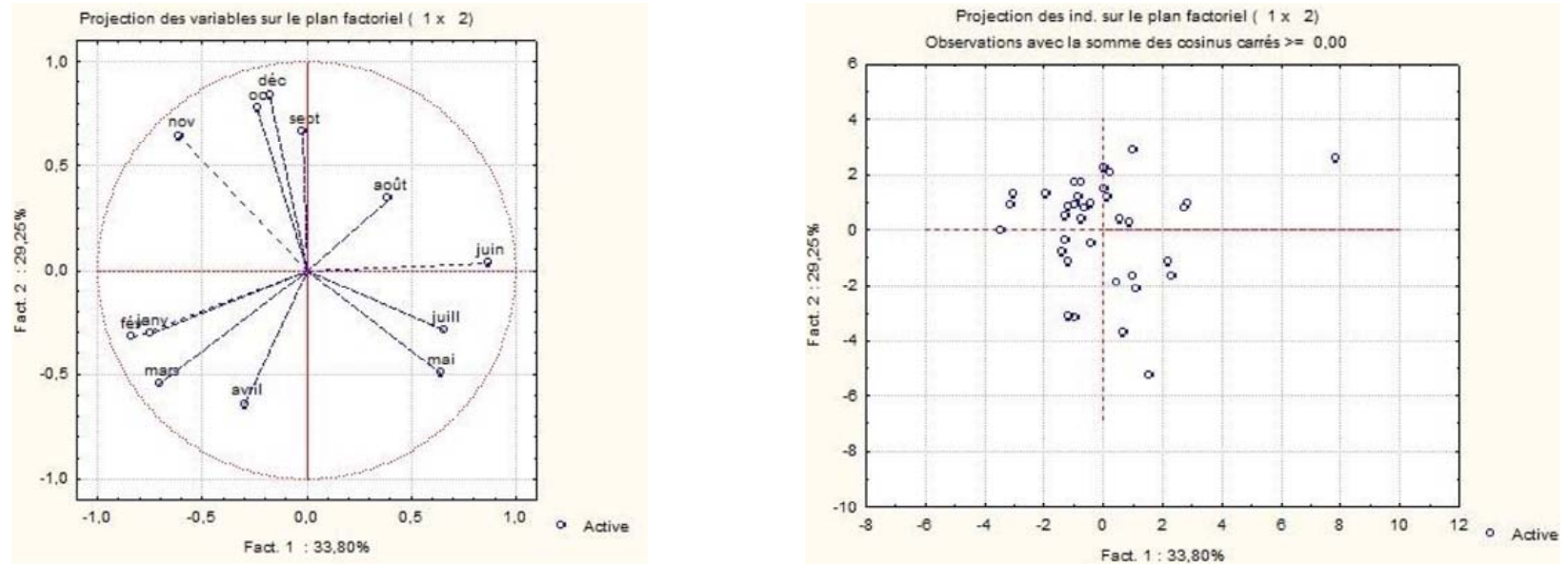

Figure 3a : Représentation et projection des variables et des exploitations laitières hors-sol $(\mathrm{n}=36)$ sur les deux premiers axes factoriels (Algérie) /// Graph and projection of variables and landless dairy farms $(n=36)$ on the first two factorial axes (Algeria)

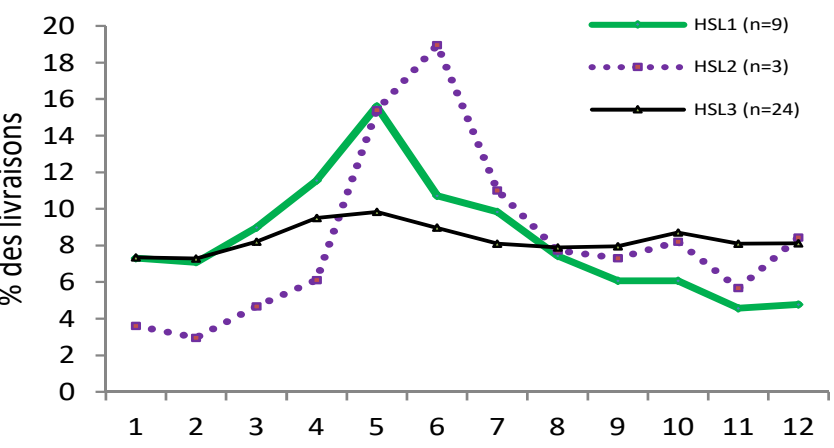

Figure $3 \mathbf{b}$ : Types de profils de livraison des exploitations laitières hors-sol selon le mois (Algérie) /// Types of delivery profiles of landless dairy farms by month (Algeria)

de l'été (19,0\% en juin) et par une période plus calme de livraisons en automne, avec une chute caractérisée par un creux en février ;

- profil HSL3, livraisons étalées $(\mathrm{n}=24)$. Ces exploitations présentaient des livraisons étalées sur toute l'année avec une variation mensuelle comprise entre $7,3 \%$ et $9,8 \%$.

Les profils des livraisons ont ensuite été regroupés en quatre profils types qualifiés selon la saison où se réalisait le maximum de livraisons de lait : livraisons fortement saisonnées de printemps (ATL1 et HSL1), qualifiées Liv1 ; livraisons moyennement saisonnées et tardives (ATL2 et HSL2), qualifiées Liv2 ; livraisons faiblement saisonnées et précoces (ATL3) qualifiées Liv3 ; livraisons étalées sur toute l'année (ATL4 et HSL3) qualifiées Liv4.

En système avec terre, le profil type des livraisons fortement saisonnées de printemps (Liv1) était le plus répandu $(31,4 \%)$, suivi par celui des livraisons étalées (Liv4) représentant 28,1 \%. En système horssol, le profil type des livraisons étalées (Liv4) était le plus répandu
(66,7 \%), suivi par celui des livraisons fortement saisonnées de printemps (Liv1) représentant 25,0\%.

En considérant l'ensemble des exploitations ( $\mathrm{n}=157)$, le profil type des livraisons étalées sur toute l'année (Liv4) était le plus répandu (36,9\%), suivi par celui des livraisons fortement saisonnées de printemps (Liv1) avec 29,9\%. Les deux autres profils types, Liv2 livraisons moyennement saisonnées et tardives, et Liv3 livraisons faiblement saisonnées et précoces, représentaient respectivement $15,9 \%$ et $17,2 \%$ de l'échantillon.

\section{Relation entre profils de livraison et profils de vêlage \\ Répartition des moyennes des livraisons et des vêlages pour l'ensemble des exploitations suivies}

Pour l'ensemble des exploitations suivies, les vêlages ont été relativement plus nombreux de janvier à avril (figure 4) avec un maximum en mars $(13,3 \%)$ puis une tendance à la baisse pour atteindre un fort minimum en septembre (5,0\%). Globalement, $51,1 \%$ des vêlages ont eu lieu durant les cinq premiers mois de l'année. Cette répartition des vêlages a expliqué les livraisons maximales de lait au printemps, c'est-à-dire dans les mois qui ont suivi les vêlages.

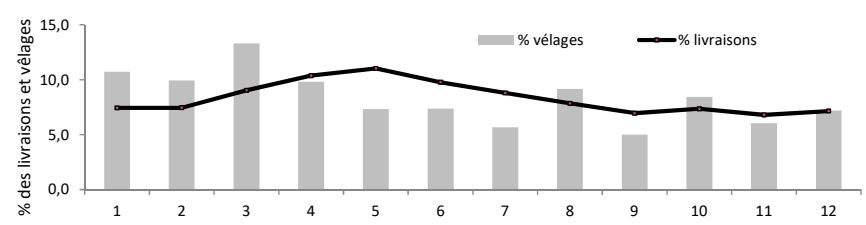

Figure 4 : Répartition des moyennes des livraisons et des vêlages de l'ensemble des exploitations laitières $(n=157)$ selon le mois (Algérie) //I Distribution of average deliveries and calvings for all dairy farms $(n=157)$ by month (Algeria) 


\section{Répartition des vêlages par profil type de livraison}

Dans le but d'expliciter les liens entre les périodes de vêlages et la répartition des livraisons de lait des exploitations, la distribution des vêlages a été analysée pour chaque profil type de livraison. Dans les exploitations du profil type de livraisons fortement saisonnées de printemps (Liv1), une concentration des vêlages en début d'année a été observée, soit $56,9 \%$ les quatre premiers mois, expliquant les livraisons maximales au printemps (figure 5). Le profil type de livraisons moyennement saisonnées et tardives (Liv2) était caractérisé par des vêlages plus tardifs. Pour ces exploitations (figure 6) les vêlages se sont concentrés au printemps (40,2\% de mars à mai), ce qui a concordé avec des livraisons maximales de lait à partir de la fin du printemps et au début de l'été (pic de 13,1\% en juin). La concentration des vêlages au printemps peut être attribuée à une synchronisation naturelle de la reproduction des troupeaux aux disponibilités fourragères. Cette concentration des vêlages entre mars et mai et le fort pourcentage de livraisons en fin de printemps et en été n'étaient pas très différents entre les deux systèmes d'exploitation. Dans le profil type de livraisons précoces et faiblement saisonnées (Liv3) observé uniquement en système avec terre où les périodes de vêlages ne sont pas planifiées, un nombre important de vêlages ont eu lieu en janvier

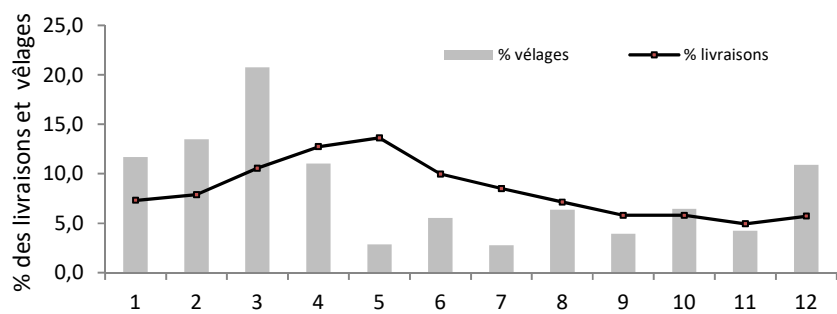

Figure 5 : Répartition des vêlages et des livraisons des exploitations laitières au profil fortement saisonné de printemps $(\mathrm{n}=47)$ selon le mois (Algérie) /// Distribution of calvings and deliveries of dairy farms with the spring profile $(n=47)$ by month (Algeria)

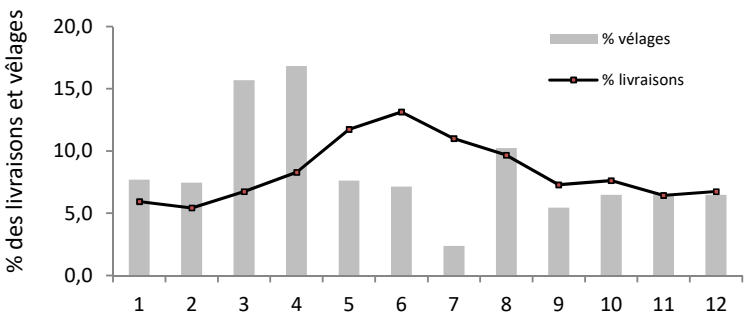

Figure 6 : Répartition des vêlages et des livraisons des exploitations laitières au profil tardif $(\mathrm{n}=25)$ selon le mois (Algérie) /// Distribution of calvings and deliveries of dairy farms with the late-season profile $(n=47)$ by month (Algeria)
(19,1 \% des vêlages) et en mars (11,3\% des vêlages) (figure 7). Cela a induit une certaine régularité des livraisons en fin d'hiver et au début du printemps. La répartition des livraisons sur toute l'année (Liv4) correspond à une répartition régulière des vêlages (figure 8). Dans ces exploitations les éleveurs avaient fait le choix de répartir les vêlages dans l'année, avec toutefois une baisse en septembre $(5,1 \%$ des vêlages) et en décembre (4,9\%).

\section{Circuits d'approvisionnement des laiteries}

\section{Capacité de collecte des laiteries}

Les laiteries A et B étaient de petites tailles et implantées à Skikda. Elles pratiquaient la collecte de lait cru tout en bénéficiant de quotas mensuels de poudre de lait subventionnée et de primes de collecte et d'intégration du lait cru. Cet accès aux poudres de lait leur est accordé dans le cadre d'une convention avec l'ONIL. Leur capacité de collecte est d'environ 30000 litres/jour avec des collecteurs privés sous contrat. Elles fabriquent du lait de consommation conditionné en sachets (LPS) et des produits laitiers. La part de ces deux laiteries représentait 43,7\% de la collecte de lait de 105 exploitations (tableau II).

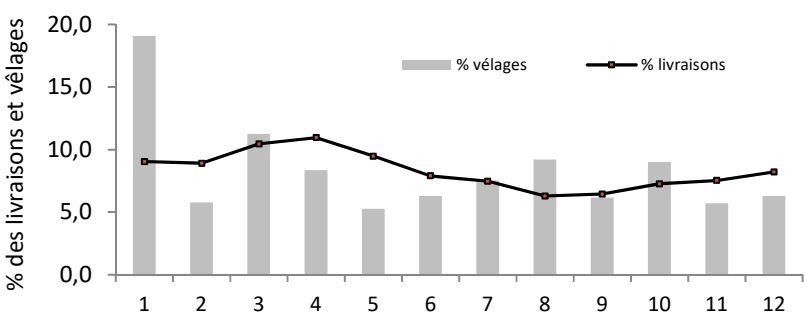

Figure 7 : Répartition des vêlages et des livraisons des exploitations laitières au profil précoce $(n=27)$ selon le mois (Algérie) // Distribution of calvings and deliveries of dairy farms with the earlyseason profile $(n=47)$ by month (Algeria)

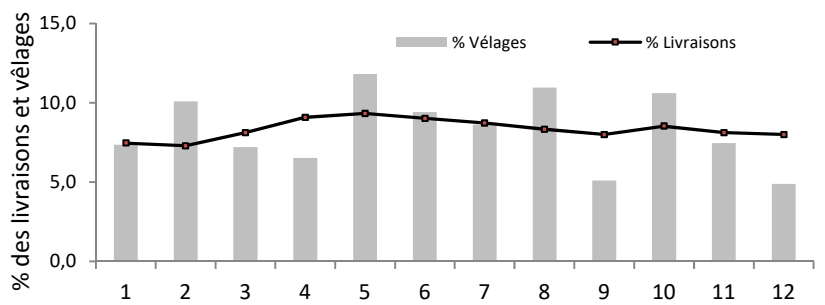

Figure 8 : Répartition des vêlages et des livraisons des exploitations laitières au profil étalé $(\mathrm{n}=58)$ selon le mois (Algérie) /// Distribution of calvings and deliveries of dairy farms with the throughoutthe-year profile $(n=47)$ by month (Algeria)

Tableau II : Répartition des flux laitiers collectés par laiterie et par wilaya (Algérie) //I Distribution of milk flows collected by dairy and by wilaya (Algeria)

\begin{tabular}{|c|c|c|c|c|c|c|}
\hline \multirow[t]{2}{*}{$\mathbf{N}$} & \multirow{2}{*}{$\begin{array}{l}\text { Production laitière } \\
\text { (x } 1000 \text { litres) }\end{array}$} & \multirow{2}{*}{$\begin{array}{c}\text { Adhésion laiterie } \\
\text { Nb. }(\%)\end{array}$} & \multirow[t]{2}{*}{ Laiterie (wilaya) } & \multirow{2}{*}{$\begin{array}{l}\text { Lait collecté } \\
\text { (x } 1000 \text { Llitres) }\end{array}$} & \multicolumn{2}{|c|}{ Lait collecté (\%) } \\
\hline & & & & & Laiterie & Wilaya \\
\hline \multirow[t]{4}{*}{157} & 2442,2 & $79(50,3)$ & A (Skikda) & 1638,6 & 32,2 & 43,7 \\
\hline & 851,3 & $26(16,6)$ & B (Skikda) & 582,6 & 11,5 & \\
\hline & 2541,9 & $37(23,6)$ & C (Вejaïa) & 1570,1 & 30,8 & 56,3 \\
\hline & 2019,0 & $15(9,5)$ & D (Вejaïa) & 1299,1 & 25,5 & \\
\hline Total & 7854,4 & $157(100)$ & & 5090,4 & 100 & 100 \\
\hline
\end{tabular}


Les laiteries $\mathrm{C}$ et $\mathrm{D}$ implantées à Bejaïa étaient des unités industrielles de grande taille avec des capacités de collecte et de production sept fois supérieures à celles des laiteries A et B. Elles pratiquaient la collecte du lait dans plusieurs wilayas pour fabriquer surtout des produits laitiers. Elles ne fabriquaient pas de LPS et n'avaient donc pas accès à de la poudre de lait subventionnée. La laiterie $\mathrm{C}$ exploitait des bassins laitiers répartis dans une plus grande zone de collecte avec 3000 producteurs de lait et 40 centres régionaux de collecte. Quant à la laiterie D, elle était approvisionnée en lait cru par 1000 producteurs à travers 36 centres de collecte répartis dans une zone moins grande que celle de la laiterie $\mathrm{C}$. Ces deux laiteries représentaient 56,3\% de la collecte de lait de 52 exploitations (tableau II). Par ailleurs, le volume global de lait était collecté dans 21 communes de Skikda où se trouvaient les 157 exploitations (figure 9).

\section{Stratégies des laiteries dans le choix des élevages laitiers}

Les petites laiteries A et B de Skikda étaient engagées dans la fabrication de lait de consommation vendu à prix règlementé grâce aux quotas de poudre de lait subventionnée. Cependant, pour la fabrication de produits laitiers vendus à prix libres sur le marché, elles utilisaient le lait cru collecté. Cela expliquait leur faible niveau d'intervention dans le soutien du développement de la production laitière locale. Les élevages qui livraient à ces deux laiteries produisaient en moyenne 4824,3 litres par vache par an, pour un nombre moyen de vaches laitières de 6,2 (laiterie A) et 6,7 (laiterie B) par exploitation (figure 10). Quant aux laiteries industrielles $\mathrm{C}$ et $\mathrm{D}$ de Bejaia, elles dépendaient du lait cru dans la fabrication exclusive de produits laitiers vendus à prix libres. Elles appliquaient une stratégie plus engagée avec les éleveurs laitiers en densifiant leurs réseaux de collecte et en garantissant un appui technique permettant de fidéliser les éleveurs et d'assurer une régularité de leur approvisionnement en lait cru. Leurs techniciens se chargeaient d'intégrer en priorité les exploitations disposant de grandes superficies agricoles et de troupeaux de grande taille.

Les laiteries $\mathrm{C}$ et $\mathrm{D}$ accordaient aux éleveurs adhérents une assistance technique pour l'entretien du matériel de traite et de réfrigération. Elles leur apportaient aussi des conseils techniques sur la conduite de la reproduction, de l'alimentation et de la traite à travers des formations organisées par des zootechniciens et des vétérinaires employés par la laiterie. Elles adoptaient la stratégie du leasing en termes de financement et d'importation de génisses pleines de races sélectionnées au profit des éleveurs et dont le remboursement des crédits se faisait par la livraison de leur production laitière à la laiterie. Les élevages adhérents de la laiterie $\mathrm{C}$ disposaient en moyenne de 10,5 vaches par exploitation et ceux adhérents à la laiterie $\mathrm{D}$ en possédaient en moyenne 18. Ces deux catégories d'éleveurs produisaient en moyenne respectivement 6205,5 litres et 7237,2 litres par vache et par an. La stratégie adoptée par les laiteries $C$ et $D$ de Bejaia appliquée aux éleveurs de Skikda a révélé un certain succès par l'accaparement de plus de $50 \%$ de la production laitière disponible en facilitant l'achat d'animaux laitiers, l'acquisition de matériels de froid et la formation du personnel technique de la ferme.

\section{Débouchés du volume de lait collecté}

Les volumes de lait produit par les exploitations étaient écoulés selon trois principaux débouchés : l'autoconsommation, les ventes du circuit formel auprès des laiteries et les ventes du circuit informel (crèmeries et clientèle). La production laitière annuelle des exploitations était de l'ordre de $7854000 \mathrm{~L}$ dont $5090000 \mathrm{~L}$ (64,8 \%) étaient livrés aux quatre laiteries. L'autoconsommation par les veaux et la famille de l'éleveur était de 789000 L (10,0\%). Cette faible part de lait cru autoconsommé témoignait de la stratégie adoptée par les éleveurs de prioriser la commercialisation. Le recours des exploitations à la vente de leur lait dans le circuit informel (cafés, crèmeries et clientèle) était dû au fait que le prix était plus rémunérateur. Ainsi, le volume de lait écoulé dans le circuit informel (crèmeries et cafés et clientèle) était environ de 1975000 L (25,2\%).

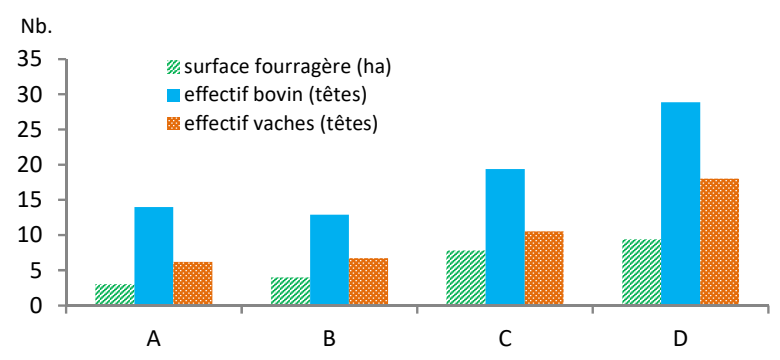

Figure 10 : Caractéristiques des exploitations selon les laiteries d'affiliation (Algérie) /// Characteristics of the farms according to the dairies of affiliation (Algeria)
Figure 9 : Répartition des flux de lait collecté par commune de la wilaya de Skikda (Algérie) III Distribution of milk flows collected by commune of the wilaya of Skikda (Algeria)

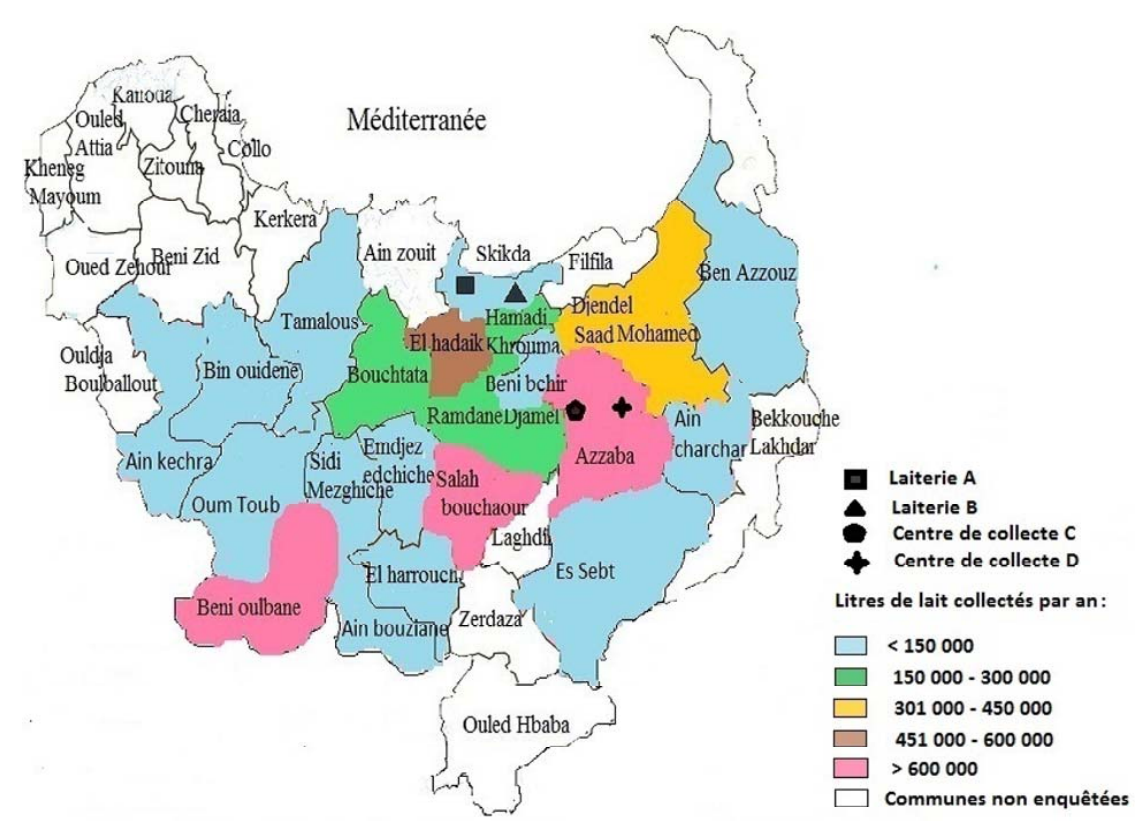




\section{Contribution des profils types de livraison dans l'approvisionnement des laiteries}

La laiterie A de Skikda était principalement approvisionnée par les exploitations des profils types de livraisons tardives, livraisons saisonnées de printemps et livraisons étalées $(27,0 \%)$ qui leur assuraient une certaine régularité dans la transformation (figure 11). La laiterie B était plutôt approvisionnée par les exploitations aux profils types de livraisons étalées sur l'année et de livraisons précoces. La sons saisonnées de printemps et livraisons précoces. La laiterie D était approvisionnée également par les exploitations de deux profils : livraisons étalées sur l'année et livraisons précoces (figure 11).

\section{Distances entre exploitations et laiteries}

La distance moyenne des exploitations avec la laiterie A était plus élevée (42 km) qu'avec la laiterie B (28 km) (figure 12). Ceci était lié au fait qu'un grand nombre d'exploitations adhérentes à la laiterie $\mathrm{A}$ étaient localisées dans la commune de Benioulben distante de plus de $50 \mathrm{~km}$. La localisation des laiteries $\mathrm{C}$ et $\mathrm{D}$ à Bejaia explique les grandes distances (plus de $250 \mathrm{~km}$ ) les séparant de leurs exploitations adhérentes (figure 12 à droite). Cependant, l'implantation de leurs deux centres relais de collecte dans le bassin laitier de Skikda réduisait relativement la distance moyenne entre elles et leurs exploitations.

\section{DISCUSSION}

\section{Une production laitière très saisonnière}

Avec un ratio moyen de saisonnalité de 1,62 pour l'ensemble des exploitations, la production laitière apparait fortement saisonnière à Skikda. Une valeur plus faible $(1,23)$ a été signalée chez des exploitations bovines laitières en Bretagne (France) par Losq et al. (2007).

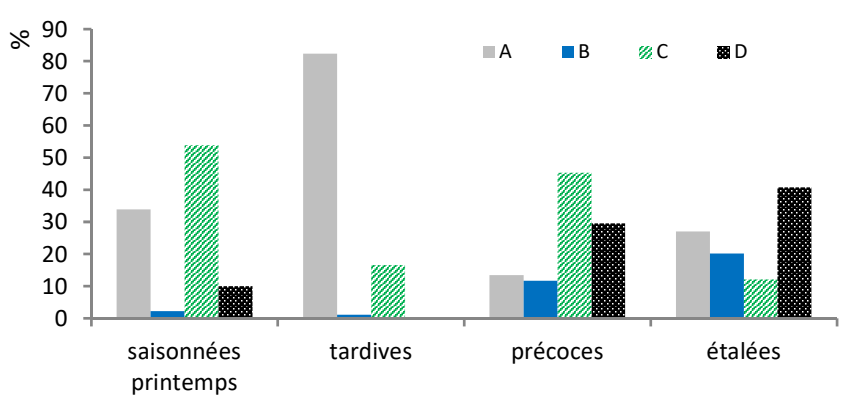

Figure 11 : Contribution selon le type de profil des exploitations dans les livraisons aux laiteries (A, B, C et D) (Algérie) /I/ Profilebased farm contribution to deliveries to dairies ( $A, B, C$ and $D$ ) (Algeria)

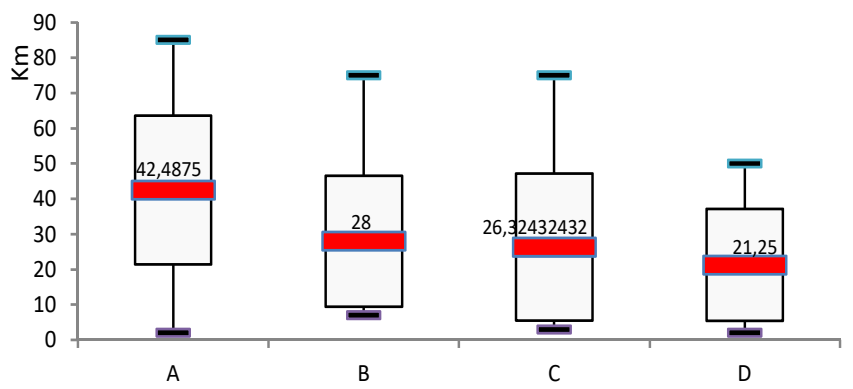
laiterie $\mathrm{C}$ de Bejaia était fournie en lait par les exploitations des livrai-

En revanche, un rapport de saisonnalité variable (1,3 à 3,7) a été également observé pour 12 profils de livraison identifiés dans une étude réalisée par le réseau d'élevage en Lorraine (Delmotte et al., 2008).

Ces irrégularités se traduisaient dans des variations saisonnières très fortes des livraisons du lait aux laiteries, limitant ainsi la participation des producteurs locaux dans les circuits de transformation, comme c'est le cas dans d'autres pays. Au Sénégal, par exemple, Dieye et al. (2005) rapportent, dans une étude sur la filière laitière périurbaine à Kolda, que les irrégularités de la production laitière compromettent les capacités des laiteries à offrir de manière régulière des produits laitiers locaux sur le marché.

La saisonnalité de la production tenait bien sûr à l'irrégularité saisonnière de la disponibilité en fourrages mais surtout à une forte saisonnalité des vêlages constatée dans les deux systèmes. Nos suivis ont souligné les différentes stratégies des éleveurs en matière de vêlage, avec une minorité d'entre eux qui faisaient le choix de répartir les vêlages (et la production) sur toute l'année. Losq et al. (2007) font le même constat dans une étude portant sur 3545 exploitations bretonnes en 2002-2004. Une autre étude (Chambre d'agriculture du Pays de la Loire, 2006) rapporte que, sur un effectif de 345 vaches laitières, $40 \%$ des vêlages ont eu lieu entre août et octobre 2004, et seulement $15 \%$ entre janvier et mars 2005.

Cela étant, chacun des profils types de livraisons (lait de printemps, lait de fin printemps-été et lait d'hiver) a joué un rôle dans l'approvisionnement des laiteries. Cependant, le profil lait étalé sur toute l'année assurait un approvisionnement important et régulier aux laiteries. En revanche, la diversité des situations semblait intéressante dans la mesure où l'approvisionnement des laiteries était maintenu au cours des quatre saisons. Selon Napoléone (2019), les systèmes d'élevage caprin avec des mises bas de printemps assurent $60 \%$ des livraisons de lait en été et début automne alors que les systèmes avec mises bas en automne assurent $60 \%$ de la collecte en automne et début hiver. Selon cette autrice, ce constat a fait évoluer le regard des acteurs sur l'intérêt de la diversité au sein du bassin de collecte de la laiterie.

Les exploitations suivies révèlent le manque de coordination au niveau de l'interprofession laitière qui n'a pas permis aux éleveurs de faire des choix sur l'orientation technique de leurs troupeaux en relation avec les laiteries auxquelles ils livraient leur lait. La réduction de la saisonnalité nécessite une concertation entre producteurs et industriels laitiers en matière de conduite de la reproduction des troupeaux (planification des dates de saillies) et d'organisation de la collecte (densification des réseaux de collecte de lait), comme suggéré par Gaye et al. (2020) dans une étude portant sur 323 élevages adhérents de la laiterie du Berger (Sénégal) : le mode de reproduction et la programmation des moments de saillie pourraient remédier à la saisonnalité de la production laitière. Dans tous les cas, la consommation de lait en Algérie est régulière toute l'année grâce à un

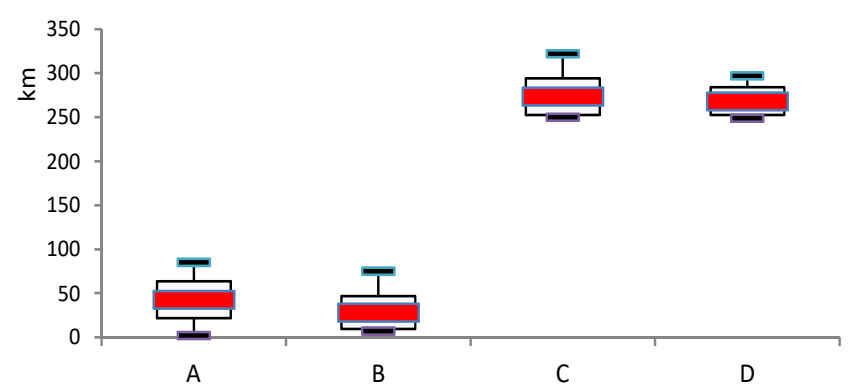

Figure 12 : Distances moyennes des exploitations laitières vis-à-vis des centres de collecte, des petites laiteries (A et B) à Skikda et des laiteries industrielles (C et D) à Bejaia (Algérie) /// Average distances between dairy farms and collection centers, small dairies (A and B) in Skikda, and industrial dairies (C and D) in Bejaia (Algeria) 
approvisionnement du marché à base de lait cru et de poudres de lait transformées par les laiteries conventionnées.

\section{Concurrence de la poudre de lait}

Depuis la relance du programme national de réhabilitation de la filière laitière locale, les politiques de soutien à la consommation de masse basées sur le LPS subventionné et la fixation du prix au détail du litre de ce lait à 25 DA ont ralenti fortement l'émergence du lait local sur le marché du lait frais, malgré les différentes mesures d'incitations financières de l'Etat mises à jour périodiquement pour appuyer le secteur laitier public défaillant et dynamiser un secteur privé émergent (Belhadia et al., 2014 ; Fares et Mamine, 2018).

Dans le contexte de Skikda, la création des laiteries A et B en 2004 n'a pas été suffisante pour drainer et intégrer le maximum de lait cru. L'implantation dans cette wilaya de deux centres régionaux de collecte approvisionnant à très grande distance les laiteries $\mathrm{C}$ et $\mathrm{D}$ de Bejaia permet, certes, d'accroître les volumes de lait collectés mais ces laiteries fabriquent exclusivement des produits laitiers vendus à prix libres. Cela ne contribue pas à réduire l'importation de poudres de lait destinées à la fabrication de LPS vendu à prix subventionné dans les centres urbains à forte démographie. Abbas et al. (2009) signalent, en outre, que du point de vue de la rentabilité des usines laitières, il y a une concurrence directe de la reconstitution du lait en poudre, opération subventionnée à l'amont, avec la production de lait de vache qui occasionne les surcoûts de qualité et de saisonnalité des livraisons de lait auxquels ne répond pas la politique des prix administrés. Les laiteries $\mathrm{A}$ et $\mathrm{B}$ pourraient accompagner les éleveurs dans l'acquisition de matériels de réfrigération (cuves réfrigérantes) qu'ils rembourseraient par les livraisons de leur production laitière, à l'instar des laiteries $C$ et D qui sont dotées de moyens matériels conséquents leur permettant de choisir et de fidéliser les producteurs laitiers performants.

\section{Enjeux en termes de politiques laitières publiques}

Les pouvoirs publics continuent à soutenir la consommation urbaine par le recours à l'importation de poudres de lait subventionnées à travers de quotas mensuels aux laiteries conventionnées à partir de 2008 dans le cadre du programme de régulation du marché par l'ONIL. Quant à la production laitière locale, elle reste faiblement intégrée industriellement (Benyoucef, 2017 ; Fares et Mamine, 2018). Le même constat a été fait au Sénégal où la consommation de lait et produits laitiers est fortement couverte par l'importation de lait en poudre alors que la collecte de la production locale reste coûteuse (Corniaux, 2015). Les expériences dans ce domaine en Afrique montrent que, s'il y a une volonté réelle de promouvoir la production locale, l'appui à la collecte doit être accompagné par la mise en place d'infrastructures de collecte en mesure d'appuyer les éleveurs en leur facilitant l'accès aux crédits bonifiés pour l'acquisition d'équipements de refroidissement et de stockage, afin de préserver la qualité du lait produit et son paiement négocié avec les industriels.

Cela conduirait à une plus forte incorporation du lait cru dans la fabrication de lait frais pasteurisé et par conséquent une limitation du recours aux poudres de lait importées très coûteuses pour l'Etat. Des mesures incitatives doivent être alors révisées par les pouvoirs publics en faveur des industriels laitiers pour les stimuler à apporter leur appui aux activités d'agroélevage en termes de formation et de transfert technologique pour la conduite des élevages et la préservation de la qualité du lait à la ferme. Il s'agit de contribuer à ce que les industriels laitiers s'approprient leurs bassins laitiers pour le développement et la diversification de la filière laitière dans son ensemble (Duteutre, 2007 ; 2019 ; Duteurtre et al., 2013).

Les laiteries industrielles $\mathrm{C}$ et $\mathrm{D}$ investissaient dans le domaine de la collecte du lait auprès d'éleveurs en leur octroyant des crédits à l'achat de génisses ou d'équipement. Elles fidélisent par ce biais des producteurs et collectent des volumes de lait importants sur tout le territoire national pour fabriquer des produits laitiers à forte valeur ajoutée tout en utilisant la poudre de lait importée. En effet, la collecte du lait local peut ainsi être intéressante pour les industriels locaux qui ont réussi à créer des liens forts avec des éleveurs dans le cadre de mesures incitatives et régulatrices comme celle signalée dans le cas de laiteries en Afrique de l'Ouest (Corniaux et Duteurtre, 2018). Dans notre étude, il s'agit des laiteries $\mathrm{C}$ et $\mathrm{D}$ en Algérie où la croissance démographique et l'exode rural vers les villes ont induit une forte demande en lait et produits laitiers. Cela a conduit les pouvoirs publics à stimuler le secteur industriel laitier à participer au programme de régulation du marché par l'ONIL. Cependant, de nombreuses questions restent posées pour identifier des moyens concrets pour encourager ces nouveaux partenariats entre laiteries et producteurs.

Le lait produit au niveau des exploitations suivies a essentiellement trois débouchés : l'autoconsommation, la vente de lait dans le circuit formel (laiteries) et la vente dans le circuit informel qui continue à absorber le quart de la production (clientèle et colportage). Ce constat est proche de celui de Djermoun et Chehat (2010) qui indiquent que le circuit informel absorbe l'équivalent de $24,53 \%$ de la production locale. Cela met en évidence la faible connexion contractuelle entre les producteurs et les industriels par manque de concertation au sein de l'interprofession de la filière laitière locale et l'inapplication de la règlementation exigeant la pasteurisation du lait cru avant sa mise sur le marché de consommation (Benyoucef et al., 2013). Cependant, il faut nuancer ce constat en faisant remarquer que dans certaines wilayas, le taux de collecte de lait par l'industrie est plus important. Ainsi, dans le cas de Tizi-Ouzou, le taux de collecte s'élève en moyenne à $90 \%$ de la production totale d'un échantillon de 83 exploitations enquêtées (Ghozlane et al., 2010). Il en résulte que le respect des engagements et des obligations unissant la laiterie à ses éleveurs adhérents permet d'intégrer industriellement le lait local collecté et de faire éviter sa déviation vers le circuit informel.

A Skikda, plus de la moitié $(56,4 \%)$ du volume collecté par l'industrie (2,9 millions de litres) était exporté hors wilaya vers les laiteries $\mathrm{C}$ et $\mathrm{D}$ de Bejaia qui, en fait, venaient en appui aux laiteries A et B de Skikda qui transformaient le reste $(43,6 \%)$. Berbiche (2019) rapporte que $48,4 \%$ du volume de lait produit dans la wilaya de Ghardaïa (188 163 L) est transféré vers les laiteries $\mathrm{C}$ et $\mathrm{D}$ de Bejaia. Par ailleurs, Akmoum et Boutaba (2017) indiquent aussi que 85,6 \% de la production de lait de la wilaya de Bordj Bou Arreridj (1,9 million de litres) est collectée et transférée vers la laiterie Hodna implantée dans la wilaya de M'Sila.

En Algérie, les laiteries doivent en principe s'orienter davantage vers les bassins laitiers de leurs espaces géographiques et renforcer le maillage de leurs propres réseaux en négociant des contrats spécifiques, à la fois avec des producteurs et des collecteurs. L'adoption par consensus de modes de fixation du prix du lait cru et des primes liées à sa qualité selon la saison inciterait probablement un grand nombre d'acteurs de la filière à rejoindre le circuit formel (Makhlouf, 2015). Les collecteurs intervenant pour les laiteries A et B de Skikda ne semblaient pas jouer pleinement leur rôle d'intermédiaires entre la laiterie et les éleveurs laitiers. Cependant, Keffache et Tozanli (2015) rapportent que les collecteurs de la wilaya de Médéa jouent un rôle dans l'arbitrage et l'ajustement des livraisons du lait vers l'industrie.

Actuellement, les pouvoirs publics tendent à valoriser davantage les produits agricoles locaux afin de limiter l'utilisation de produits importés coûteux comme la poudre de lait, notamment dans une période où les ressources financières s'amenuisent. Au Burkina Faso et au Sénégal, Broutin et al. (2018) signalent que les importations de lait exercent une concurrence très forte sur le lait local. Cela nécessite de raisonner et d'adapter des mesures de soutien à la filière laitière locale combinant des actions de politique agro-industrielle participative au niveau de 
la production et de la transformation, en pesant sur la compétitivitéprix relative entre produits importés et produits locaux, et en menant une offensive de promotion des produits à base de lait local auprès des consommateurs. Une collecte de lait mieux organisée et performante permettrait d'établir des liens durables et fidélisés entre la production et la transformation, et de favoriser le développement de la filière.

\section{CONCLUSION}

L'émergence de filière laitière locale nécessite une densification des réseaux de collecte qui doivent s'adapter aux systèmes d'élevage pratiqués dans chaque zone de production de lait. Les effectifs bovins de races locales et croisées constituent plus des trois quarts du cheptel bovin de la wilaya de Skikda. Ce type de cheptel mérite d'être intégré dans le développement de la filière lait pour la fabrication de lait et de produits laitiers d'appellation traditionnelle (Dhen, klila, lben et autres) à l'instar de ce qui se fait ailleurs en Algérie.

En 2017, la production laitière de Skikda a été estimée à 126,76 millions de litres faiblement intégrés par l'industrie, notamment celle d'exploitations détenant de vaches de races laitières localisées principalement dans les communes de Benioulben, Ramdane Djamel, Azzaba, Salah Bouchaour et Bouchtata où le potentiel de production de lait livrable est le plus élevé. La stratégie à appliquer pour ce système d'élevage consisterait à valoriser le lait produit en encourageant les éleveurs motivés par un appui technique régulier qui les inciterait à s'intégrer dans les réseaux de collecte formels durables. Ceci permettrait de satisfaire pleinement les besoins en lait des unités laitières et de valoriser la production locale tout en garantissant la commercialisation du lait à un prix rémunérateur pour les producteurs.

Le soutien par les pouvoirs publics à la création d'organisations d'éleveurs et d'unités de transformation du lait cru au niveau des différentes zones (montagnes et plaines) de Skikda constituerait l'une des solutions pour le développement intégré des trois segments de la filière laitière locale (production, collecte et transformation). Du point de vue de l'industriel, la contrainte de la saisonnalité de l'approvisionnement des laiteries en lait local peut être atténuée par la densification des réseaux de collecte et la fabrication de lait de longue conservation pouvant répondre ainsi à la demande de la population en saison sèche (automne) et absorber la forte production printanière. Cela suppose que toute la production laitière de la ferme transite par le circuit formel.

L'analyse de la répartition des vêlages a mis en évidence que la plupart des éleveurs nécessitaient un appui technique pour mieux maitriser la conduite de la reproduction de leurs troupeaux afin de programmer les périodes de livraisons de lait aux laiteries. Afin d'éviter toute pénurie de lait conditionné en sachets dans les centres urbains, les pouvoirs publics ont finalement pris une mesure récente (décret exécutif $n^{\circ} 20-153$ du 8 juin 2020) orientant ce type de lait subventionné exclusivement aux ménages et l'interdisant à tout agent économique (établissements de débits de boissons, cafés et restaurants). Cette mesure pourrait-elle induire plus d'intérêt de la part des industriels vers les bassins laitiers des wilayas pour collecter et utiliser exclusivement le lait cru dans leur fabrication de lait et de produits laitiers locaux ?

\section{Remerciements}

Les auteurs remercient les responsables des laiteries, des centres de collecte de lait et des élevages suivis pour leur accueil et leur contribution dans la réalisation de cette étude. Les auteurs remercient également Martine Napoléone pour ses commentaires sur le manuscrit.

\section{Conflits d'intérêts}

L'étude a été réalisée sans conflit d’intérêts.

\section{Déclaration des contributions des auteurs}

KZ a contribué à la réalisation de l'étude par la délimitation et le suivi des élevages bovins et les enquêtes auprès des quatre laiteries ; elle a participé à la validation des questionnaires à renseigner en entreprise (élevages et laiteries) ; elle a contribué aux analyses statistiques et à l'interprétation des résultats ; elle a participé à la rédaction et à la révision du manuscrit. GD a contribué à la conception et à l'enrichissement du manuscrit ; il a participé à l'interprétation des résultats d'analyses et a contribué à la rédaction et à la révision du manuscrit. MTB a contribué à la conception et à la coordination de l'étude sur la filière laitière ; il a participé à l'élaboration des questionnaires de suivi des élevages bovins et d'enquêtes auprès des laiteries ; il a contribué à l'interprétation des résultats d'analyses statistiques, à la rédaction et à la révision du manuscrit.

\section{REFERENCES}

Abbas K., Riahi O., Madani T., 2009. Les filières laitières de la région Algérienne de Sétif : Diversité et contraintes. Rev. Rencontres Rech. Rumin., 16

Akmoum H., Boutaba H., 2016. La filière lait cru dans la wilaya de Bordj Bou Arreridj : Analyse des caractéristiques des livraisons de lait cru à l'industrie de transformation. Mém. Master ENSA, Alger, Algérie, 71 p.

Belhadia M., Yakhlef H., Bourbouze A., Djermoun A., 2014. Production et mise sur le marché du lait en Algérie, entre formel et informel : stratégies des éleveurs du périmètre irrigué du Haut-Cheliff. New Medit, CIHEAMIAMB, 13 (1): 41-49 hal.archives-ouvertes.fr/hal-02163626

Benyoucef M.T., 2005. Diagnostic systémique de la filière lait en Algérie organisation et traitement de l'information pour l'analyse des profils de livraison en laiteries et des paramètres de production des élevages. Thèse Doct., Institut National d'Agronomie, Alger, Algérie, 237 p.

Benyoucef M.T., Omari C., Amiali M., Berkani M.L., Kaci F., Benjemaa O. 2013. Formation universitaire en agronomie et introduction d'innovations techniques dans l'agro-industrie. Cas de la filière lait cru en Algérie. Conf. Int. Les systèmes d'innovation et le nouveau rôle des universités (COSINUS). ENP, Oran, Algérie

Benyoucef M.T., 2017. Filières lait et viandes : Analyses des contraintes de production et des perspectives d'intégration industrielle. Projet CNPRU Laboratoire d'économie agricole, agro-alimentaire et rurale, ENSA, Alger, Algérie, 2014-2017

Berbiche M.L., 2019. Caractéristiques de la production laitière d'exploitation bovines enquêtées dans la wilaya de Ghardaïa. Mém. Master ENSA Alger, Algérie, $70 \mathrm{p}$.

Broutin C., Levard L., Goudiaby M.C., 2018. Quelles politiques commerciales pour la promotion de la filière « lait local » en Afrique de l'Ouest, Rapport de synthèse, Gret, Paris, France, 100 p.

Chambre d'Agriculture du Pays de la Loire, 2006. Saisonnalité des livraisons de lait bio en Pays de la Loire. pays-de-la-loire.chambresagriculture.fr/ fileadmin/user_upload/National/FAL_commun/publications/Pays_de_la_ Loire/22-2006_lait_saisonnalite.pdf

Corniaux C., 2015. Bassin laitier de la basse vallée du fleuve Sénégal (Sénégal). Le développement de la filière entre lait local et lait en poudre importé. In : Napoléone M., Corniaux C., Leclerc B. (eds.) Voies lactées. Dynamiques des bassins laitiers entre globalisation et territorialisation. Cardère, Avignon, France, 143-155

Corniaux C., Duteurtre G., 2018. Pour une alliance renouvelée entre industriels et éleveurs laitiers en Afrique de l'Ouest. Oxford, OXFAM, 8 p. (Note d'orientation)

Delmotte E., Albert M., Leclair A., Pechey B., Zsitko B., Caillaud D., 2008. Saisonnalité de la production laitière bovine en région Lorraine. Etat des lieux dans les élevages. Nouvelles répartitions des vêlages, Interprofession laitière régionale Lorraine (CIRELL), $32 \mathrm{p}$.

Dieye P.N., Duteurtre G., Sissokho M.M., Sall M., Dia D., 2005. Linking local production to urban demand: the emergence of small-scale milk processing units in Southern Senegal. Livest. Res. Rural Dev. 17: 40

Dieye P.N., Montaigne E., Duteurtre G., Boutonnet J.P., 2008. Le rôle des arrangements contractuels dans le développement du système laitier local et des mini-laiteries au Sénégal. Econ. Rurale, 303-305: 108-122, doi: 10.4000/economierurale.554

Djermoun A., Chehat F., 2010. Les circuits empruntés par le lait local dans le Chéliff en Algérie : importance du circuit informel. Livest. Res. Rural Dev. 22: 199 
Duteurtre G., 2007. Trade and development of dairy production in West Africa: a Review. Rev. Elev. Med. Vet. Pays Trop., 60 (1-4): 209-223, doi: 10.19182/remvt.9972

Duteurtre G., Corniaux C., Dao D., Dia D., Mama Sambo A., Vias G.F., Cesaro J-D., 2013. Etude relative à la formulation d'un programme d'actions détaillé de développement de la filière lait au sein de I'UEMOA : Rapport définitif. CIRAD, Montpellier, France, 106 p. agritrop.cirad.fr/571594/

Duteurtre G., 2019. Les produits laitiers africains à l'épreuve de la libéralisation : Des traditions laitières en danger, un patrimoine à valoriser. $3^{\text {es }}$ Rencontres internationales sur le lait, vecteur de développement, Dakar, Sénégal, 12-13 juin 2019, agritrop.cirad.fr/594387/1/ID594387.pdf

Fares M., Mamine F., 2018. Les choix contractuels dans le secteur laitier algérien : entre spécificité des actifs et régulation publique. Can. Sci. Reg. 41 (1/3): 1-14

FAO (2020). Statistiques agricoles. FAO, Rome, Italie

Gaye P.A.M., Traoré E.H., Cisse M., Dieng A., Cesaro J.-D., Sall C., 2020. Typologie des systèmes bovins du bassin de collecte de la Laiterie Du Berger (LDB). Anim. Plant. Sci. 44 (1): 7577-7590, doi: 10.35759/JAnmPISci. v44-1.4

Ghozlane F., Belkheir B., Yakhlef H., 2010. Impact du Fonds National de Régulation et de Développement Agricole sur la durabilité du bovin laitier dans la wilaya de Tizi-Ouzou (Algérie). New Médit 9 (3): 22-27
Hadji Kouidri H., Harrache B., Ben Amirouche H., 2018. Analyse structurelle de la filière lait en Algérie. Rev. Econ. Nord. Afr., 14 (19): 39-47, doi: 10.33858/0470-000-019-027

Kheffache H., Tozanli S., 2015. La collecte de lait dans la wilaya de Médéa : Conventions et formes de coordination. 9e Journées de Recherches en Sciences Sociales 10-11 Déc 2015, Nancy, France, 16 p.

Losq G., 2007. Les livraisons mensuelles de lait en Bretagne. Une répartition des vêlages souvent subie. Cap Elev. 15: 4-6

Losq G., Espinasse R., Queffelec A., Trou G., Grasset M., Le Lan B., 2007. Saisonnalité de la production laitière en Bretagne : situation actuelle et impact des périodes de livraison sur les résultats économiques d'une exploitation laitière. Rencontres. Rech. Rumin. 14

Makhlouf M., 2015. Performance de la filière laitière locale par le renforcement de la coordination contractuelle entre les acteurs : Cas de la wilaya de Tizi-Ouzou-Algérie Thèse Doct., Université Mouloud Mammeri de Tizi-Ouzou, Algérie, 345 p.

Napoléone M., 2019. Intermediate objects to help coordination between breeders and dairies. Example of a goat dairy cooperative in Southern France. Rev. Elev. Med. Vet. Pays Trop. 72 (2): 55-63, doi: 10.19182/ remvt. 31746

Sraïri M.T., Benyoucef M.T., Kraiem K., 2013. Les filières laitières en Afrique du Nord (Algérie, Maroc et Tunisie) : des options d'autosuffisance à la dépendance alimentaire ? SpringerPlus. 2: 162. doi: 10.1186/2193-1801-2-162

\section{Summary}

Zalani K., Duteurtre G., Benyoucef M.T. Seasonality of bovine milk production and implications for the reinforcement of industrial collection in Skikda Wilaya (Algeria)

A study of the dairy sector in the wilaya of Skikda in Algeria analyzed the seasonality of milk production on 157 cattle farms and the implications for milk collection by the industry. The surveys covered the two production systems (on land and landless) in 2016 and 2017 to assess the importance of the contributions of the farming systems in supplying dairies. A first principal component analysis identified four delivery profiles. A second analysis carried out on the calvings observed in the herds of the farms showed some agreement between the seasonality of milk deliveries and the distribution of herd calvings. The study also addressed the concepts of territories and distances traveled for milk deliveries to dairies. Some farms delivered their milk to two dairies located in Skikda, which also used a supply of milk powder at subsidized prices. Others delivered their milk to milk collection centers located in the dairy basin and belonging to two industrial dairies located in Bejaia Wilaya more than 250 kilometers away on average. These dairies were also supplied with milk powder but at free market prices. They implemented support systems for farmers that enabled them to diversify their supply in order to smooth the quantities collected and to compensate for the individual seasonality of the farms. Consultation mechanisms would enable these manufacturers to support farmers with more explicit strategies for de-seasoning production.

Keywords: cattle, raw milk, dairy farms, profiles, dairy industry, farming system, Algeria

\section{Resumen}

Zalani K., Duteurtre G., Benyoucef M.T. Estacionalidad de la producción lechera bovina e implicaciones para el refuerzo de la recolección industrial en la wilaya de Skikda (Argelia)

Un estudio sobre la filial lechera en la wilaya de Skikda (Argelia) permitió analizar la estacionalidad de la producción lechera en 157 explotaciones bovinas y las implicaciones relativas a la recolección de leche por parte de la industria. Las encuestas se centraron sobre los dos sistemas de producción (con tierra y fuera del terreno) en 2016 y 2017 para evaluar la importancia de las contribuciones de los sistemas de explotación en el abastecimiento de las lecherías. Un primer análisis por componente principal evidenció cuatro perfiles de distribución. Un segundo análisis efectuado sobre los partos observados en los hatos de las explotaciones evidenció una cierta concordancia entre la estacionalidad de las distribuciones de leche y la repartición de los partos de los hatos. El estudio abordó también las nociones de territorios y de distancias recorridas para las entregas de leche a las lecherías. Ciertas explotaciones entregaban su leche a dos lecherías implantadas en Skikda, las cuales también tenían acceso a un suministro de leche en polvo a precios subvencionados. Otras enviaban la leche a centros de colecta de leche implantados en la cuenca lechera y pertenecientes a dos lecherías industriales localizadas a más de 250 kilómetros en promedio en la wilaya de Behaia. Estas últimas se suministraban también con leche en polvo, pero a precios libres del mercado. Estas ponían en funcionamiento dispositivos de apoyo a los criadores que permitían diversificar su abastecimiento, con el fin de moderar las cantidades recolectadas y de compensar la estacionalidad individual de las fincas. Dispositivos de consejo podrían permitir a estos industriales el acompañamiento de los criadores en las estrategias más explicitas de anti estacionalidad de la producción.

Palabras clave: ganado bovino, leche cruda, granjas lecheras, perfil, industria lechera, sistemas de explotación, Argelia 